\title{
Prevalence of diabetic retinopathy in screening-detected diabetes mellitus: results from the Gutenberg Health Study (GHS)
}

\author{
Katharina A. Ponto ${ }^{1,2}$ • Jochem Koenig ${ }^{3}$ - Tunde Peto ${ }^{4}$ Julia Lamparter ${ }^{1}$. \\ Philipp Raum $^{1}$ • Philipp S. Wild ${ }^{2,5,6}$ - Karl J. Lackner ${ }^{7}$ • Norbert Pfeiffer ${ }^{1}$ • \\ Alireza Mirshahi ${ }^{1,8}$
}

Received: 29 April 2016 / Accepted: 18 May 2016 / Published online: 17 June 2016

(C) Springer-Verlag Berlin Heidelberg 2016

\begin{abstract}
Aims/hypothesis Individuals with type 2 diabetes mellitus may experience an asymptomatic period of hyperglycaemia, and complications may already be present at the time of diagnosis. We aimed to determine the prevalence of diabetic retinopathy in patients with newly diagnosed (screeningdetected) type 2 diabetes.

Methods The Gutenberg Health Study is a population-based study with 15,010 participants aged between 35 and 74 years. We determined the weighted prevalence of diabetic retinopathy by assessing fundus photographs. Screeningdetected type 2 diabetes was defined as an $\mathrm{HbA}_{1 \mathrm{c}}$ concentration of $6.5 \%(47.5 \mathrm{mmol} / \mathrm{mol})$ or more, no medical
\end{abstract}

Katharina A. Ponto

katharina.ponto@unimedizin-mainz.de

1 Department of Ophthalmology, University Medical Center Mainz, Langenbeckstrasse 1, 55131 Mainz, Germany

2 Center for Thrombosis and Hemostasis, University Medical Center, Mainz, Germany

3 Institute of Medical Biostatistics, Epidemiology and Informatics, Division of Biostatistics and Bioinformatics, University Medical Center, Mainz, Germany

4 NIHR Biomedical Research Centre, Moorfields Eye Hospital NHS Foundation Trust and UCL Institute of Ophthalmology, London, UK

5 Preventive Cardiology and Preventive Medicine, Department of Medicine II, University Medical Center, Mainz, Germany

6 DZHK (German Center for Cardiovascular Research), Partner Site Rhine Main, Mainz, Germany

7 Institute of Clinical Chemistry and Laboratory Medicine, University Medical Center, Mainz, Germany

8 Dardenne Eye Hospital, Bonn-Bad Godesberg, Germany diagnosis of diabetes and no intake of insulin or oral glucoselowering agents.

Results Of 14,948 participants, 1377 (9.2\%) had diabetes mellitus. Of these, 347 (25.2\%) had newly diagnosed type 2 diabetes detected by the screening. Overall, the weighted prevalence of screening-detected type 2 diabetes was $2.1 \%$. Fundus photos were evaluable for 285 (82.1\%) participants with newly diagnosed diabetes. The weighted prevalence of diabetic retinopathy in screening-detected type 2 diabetes was $13.0 \% ; 12 \%$ of participants had a mild non-proliferative diabetic retinopathy and $0.6 \%$ had a moderate nonproliferative diabetic retinopathy. Diabetic retinopathy was proliferative in $0.3 \%$. No cases of severe non-proliferative diabetic retinopathy or diabetic maculopathy were found. Thirty (14.9\%) of 202 and six (7.2\%) of 83 individuals with and without concomitant arterial hypertension, respectively, had diabetic retinopathy (OR 2.54, 95\% CI 1.06, 7.14). Visual acuity did not differ between individuals with and without diabetic retinopathy .

Conclusions/interpretation In this large European study, the prevalence of diabetic retinopathy in screening-detected type 2 diabetes was $13 \%$. Only a very small proportion of participants with detected diabetic retinopathy needed treatment.

Keywords Diabetes mellitus · Epidemiology · Maculopathy · Microangiopathy $\cdot$ Retinopathy
Abbreviations
ADDITION Anglo-Danish-Dutch Study of Intensive Treatment in People with Screen Detected Diabetes in Primary Care
GHS Gutenberg Health Study 


\section{Introduction}

The rise in prevalence of diabetes mellitus, especially type 2 diabetes, is a global health and economic problem. Diabetic retinopathy is a frequent complication of the disease and the leading cause of blindness among working-age populations in the developed world [1]. Type 2 diabetes is characterised by a long, often asymptomatic, period of hyperglycaemia. Therefore, end-organ damage, such as diabetic retinopathy, may already be present in individuals who are unaware that they have type 2 diabetes, and the likelihood of this increases with the duration of the diabetes $[2,3]$.

The analysis of retinal vessels provides the opportunity to observe early functional and morphological changes in the microvasculature before clinically significant microvascular and macrovascular complications arise [4]. Most guidelines recommend annual screening for diabetic retinopathy, but limited resources and the slow progression of diabetic retinopathy suggest that longer recall intervals should be considered if patients have no detectable lesions [5]. Screening procedures have advantages and disadvantages that must be considered $[3,4,6]$. It has been shown that healthcare costs increase with the severity of diabetic retinopathy, which suggests that preventing its progression may lower healthcare expenditure [7]. In addition, information technology based on epidemiological data may facilitate an individualised determination of screening intervals for diabetic retinopathy [8].

Models to determine a personalised screening frequency for diabetic retinopathy have been developed to help to reduce healthcare use and the costs of diabetes care. These models still require refinement as, for example, in a model proposed by van der Haijden et al, almost $12 \%$ of participants had developed sight-threatening diabetic retinopathy before the model-based time of screening [9]. Other outcome measures of screening procedures are psychosocial well-being and cardiovascular morbidity and mortality. In a targeted screening programme, the diagnosis of type 2 diabetes had no substantial adverse or positive effect on psychological well-being and perceived health status [10]. In summary, in contrast to macrovascular complications, some microvascular events, such as background retinopathy, could theoretically be prevented by earlier diagnosis and better glycaemic control [11]. Therefore, screening for diabetes may lead to the detection of retinopathy at an earlier stage before clinically evident complications occur. In line with this, it has been shown that patients who do not attend diabetic eye screenings are at increased risk of developing sight-threatening diabetic retinopathy [12].

We therefore aimed to determine the prevalence of diabetic retinopathy in persons with type 2 diabetes that was newly detected within the framework of the population-based Gutenberg Health Study (GHS). Visual acuity served as a test for functional limitations caused by diabetic retinopathy. As micro- and macrovascular events have been shown to be related to age, sex, $\mathrm{HbA}_{1 \mathrm{c}}$ concentration, hypertension and dyslipidaemia [1, 13-15], we also analysed associations between these variables and diabetic retinopathy in screening-detected type 2 diabetes.

\section{Methods}

The GHS is a population-based, prospective, observational cohort study from midwestern Germany with a total of 15,010 participants aged between 35 and 74 years. Participants in the study undergo a standardised protocol that includes ophthalmic and complete general medical examinations focused on cardiovascular variables. The detailed protocol has previously been described [16].

Fundus images were taken by trained personnel at the GHS centre using a non-mydriatic fundus camera (Visucam PRO NMTM, Carl Zeiss, Jena, Germany) in a darkened room with the pupil's natural width. Three photographs were taken of each eye at $30^{\circ}$ and $45^{\circ}$ centred on the optic nerve, and at $30^{\circ}$ centred on the macula. These images were evaluated by two certified graders (P. Raum, J. Lamparter) at the Reading Centre at Moorfields Eye Hospital, London, UK, using a specifically designed work station. The fundus images were the basis upon which we diagnosed whether diabetic retinopathy was present, and if it was, its stage. Grading of the fundus photographs was performed in line with the recommendations of the Early Treatment Diabetic Retinopathy Study.

In the present study, the term 'screening-detected diabetes' referred to screening of the general population for diabetes mellitus. Screening for type 2 diabetes in the GHS cohort was based on $\mathrm{HbA}_{1 \mathrm{c}}$ concentrations, with a cut-off value of $6.5 \%(47.5 \mathrm{mmol} / \mathrm{mol})$ as recommended by the ADA $[16,17]$. In line with this cut-off for the identification of individuals with type 2 diabetes, Sabanayagam et al found that cut-off points between $6.6 \%(48.6 \mathrm{mmol} / \mathrm{mol})$ and $7.0 \%$ $(53.0 \mathrm{mmol} / \mathrm{mol})$ were also best for identifying individuals with mild and moderate retinopathy [18]. Exclusion criteria were a medical diagnosis of diabetes by a physician and/or the intake of insulin or oral glucose-lowering agents. Cardiovascular risk factors and visual acuity (in logMAR) were measured as previously reported [17].

For the current analyses, we used data on obesity, dyslipidaemia and arterial hypertension, assessed as follows. Arterial hypertension was diagnosed if antihypertensive drugs were being taken or if a mean systolic (and/or diastolic) blood pressure of $\geq 140(\geq 90) \mathrm{mmHg}$ was recorded from the second and third standardised measurements after resting. Diabetes was diagnosed if there was a definite diagnosis and treatment by a physician, a blood glucose level $\geq 7 \mathrm{mmol} / \mathrm{l}$ after 
overnight fasting ( $\geq 8 \mathrm{~h}$ ) or $\geq 11.1 \mathrm{mmol} / \mathrm{l}$ after a fasting period of less than $8 \mathrm{~h}$. Obesity was defined as a BMI $\geq 30 \mathrm{~kg} / \mathrm{m}^{2}$. Dyslipidaemia was defined as a definite diagnosis of dyslipidaemia by a physician or an LDL:HDL-cholesterol ratio of $\geq 3.5$.

Statistical analyses We performed statistical analyses using Statistical Analysis System (SAS) software, Version 9.4 of the SAS System for Windows (SAS Institute, Cary, NC, USA). Weighting for the age and sex distributions of the local population was based on census data as of 31 December 2009, coinciding approximately with the midpoint of our recruitment phase. As the distribution of missing values (arising from fundus photographs of insufficient quality) was not age- and sex-independent, weighting was conducted without these data.

Binary logistic regression analyses indicating ORs and 95\% CIs were performed to assess age- and sex-adjusted associations between diabetic retinopathy and cardiovascular risk factors and diseases. We used a $\chi^{2}$ test to assess the associations between categorical variables, and a MannWhitney $U$ test for association analyses between binary and metric variables. Because of the small sample size, we did not perform Bonferroni's correction or multivariable analyses. Univariable associations with a two-sided $p<0.05$ were considered statistically significant.

\section{Results}

Fundus photographs of sufficient quality were available for $14,948(99.6 \%)$ of the participants. Overall, 1377 (9.2\%) had type 2 diabetes. Of these, 25.2\% (347/1377) had newly diagnosed type 2 diabetes (screening-detected disease). The weighted prevalence for screening-detected type 2 diabetes was $2.1 \%(n=306$; women [ $n=127], 1.7 \%$; men [ $n=179]$, $2.4 \%)$.

The fundus photographs of at least one eye from 285 $(82.1 \%)$ of these 347 participants were eligible for analysis. The prevalences of the various stages of diabetic retinopathy in these participants are listed in Table 1. The weighted prevalence of diabetic retinopathy (all stages) was $13.0 \%$ (12.1\% in men and $14.2 \%$ in women). Twelve per cent of participants had a mild non-proliferative diabetic retinopathy, and $0.6 \%$ a moderate non-proliferative diabetic retinopathy. Diabetic retinopathy was proliferative in $0.3 \%$ of the individuals.

The median $\mathrm{HbA}_{1 \mathrm{c}}$ was $6.8 \%(50.8 \mathrm{mmol} / \mathrm{mol})$ in both individuals with (range $6.5-15.0 \%$ [47.5-140.4 mmol/mol]) and without (range 6.5-13.8\% [47.5-127.3 mmol $/ \mathrm{mol}]$ ) diabetic retinopathy $(p=0.228)$. Overall, 202/285 $(70.9 \%)$ participants with screening-detected type 2 diabetes had arterial hypertension. Obesity and dyslipidaemia were found in $51.4 \%(146 / 284$; missing data in $n=1)$ and $43.8 \%$ (124/283; missing data in $n=2)$ of those with screeningdetected type 2 diabetes. The (age- and sex-adjusted) associations between these cardiovascular risk factors and the presence of diabetic retinopathy are summarised in Table 2.

The median visual acuity (logMAR) of both eyes was 0.00 (range: left eye -0.1 to +0.5 , right eye -0.1 to +0.7 ) in participants with diabetic retinopathy, and 0.10 (range: left eye -0.1 to +3.0 , right eye -0.3 to +2.0 ) in those without it $(p=0.16)$.

\section{Discussion}

This large European population-based study reports on the prevalence of diabetic retinopathy in individuals with diabetes mellitus newly detected from a screening examination. Furthermore, we assessed the associations of arterial hypertension, dyslipidaemia, obesity, and visual acuity with diabetic retinopathy in this subgroup of people with type 2 diabetes.

\section{Prevalence of screening-detected diabetic retinopathy In} the present study of a mid-European, west-German cohort, one in 50 participants and one quarter of all diabetic individuals were newly diagnosed as having type 2 diabetes. In this subgroup, there were 1.5 times more men than women. Approximately $13 \%$ of those with screening-detected type 2 diabetes had signs of diabetic retinopathy. Nevertheless, mostly mild forms of non-proliferative diabetic retinopathy were found in these subgroups. Only one person had proliferative diabetic retinopathy, and no case of severe non-proliferative or diabetic maculopathy was found. This means that very few patients from those newly diagnosed with diabetes in a screening setting had stages of diabetic retinopathy that needed treatment.

Until now, no data from European population-based studies on this topic have been published. Nevertheless, the present results are in line with those from studies that have used targeted screening procedures or assessed the prevalence of diabetic retinopathy in patients who were newly diagnosed in general practice. In a European population-based targeted screening procedure, the prevalence of diabetic retinopathy in screening-detected type 2 diabetes was $7.6 \%$, compared with $1.9 \%$ in patients who were newly diagnosed in general practice [19]. In the Anglo-Danish-Dutch Study of Intensive Treatment in People with Screen Detected Diabetes in Primary Care (ADDITION), patients with screen-detected diabetes had a low prevalence $(6.8 \%)$ of diabetic retinopathy and no vision-threatening lesions. The ADDITION study from Denmark was the first to describe the prevalence of diabetic retinopathy in a population of newly diagnosed type 2 diabetic patients in general practitioners' practices who were 
Table 1 Prevalence of diabetic retinopathy in diabetic individuals detected by screening

\begin{tabular}{|c|c|c|c|c|c|c|c|c|}
\hline \multirow[t]{4}{*}{ Group } & \multirow{3}{*}{\multicolumn{2}{|c|}{$\begin{array}{l}\text { Total } \\
\text { (all stages) }\end{array}$}} & \multicolumn{6}{|c|}{ Stages of diabetic retinopathy } \\
\hline & & & \multicolumn{4}{|c|}{ Non-proliferative } & \multirow{2}{*}{\multicolumn{2}{|c|}{ Proliferative }} \\
\hline & & & \multicolumn{2}{|l|}{ Mild } & \multicolumn{2}{|l|}{ Moderate } & & \\
\hline & $\%(n)$ & $95 \% \mathrm{CI}$ & $\%(n)$ & $95 \% \mathrm{CI}$ & $\%(n)$ & $95 \% \mathrm{CI}$ & $\%(n)$ & $95 \% \mathrm{CI}$ \\
\hline \multicolumn{9}{|l|}{ Total $(n=285)$} \\
\hline Study sample ${ }^{\mathrm{a}}$ & $12.6(36)$ & $9.00-17.06$ & $11.6(33)$ & $8.13-15.93$ & $0.7(2)$ & $0.09-2.52$ & $0.4(1)$ & $0.09-1.95$ \\
\hline Study population ${ }^{\mathrm{b}}$ & $13.0(32.8)$ & $9.15-17.64$ & $12.0(30.3)$ & $8.31-16.61$ & $0.6(1.4)$ & $0.05-2.31$ & $0.3(0.8)$ & $0.01-1.92$ \\
\hline \multicolumn{9}{|l|}{$\operatorname{Men}(n=171)$} \\
\hline Study sample ${ }^{a}$ & $11.7(20)$ & $7.29-17.48$ & $10.5(18)$ & $6.36-16.13$ & $1.2(2)$ & $0.14-4.16$ & $0.6(1)$ & $0.01-3.22$ \\
\hline Study population ${ }^{\mathrm{b}}$ & $12.1(18.1)$ & $7.41-18.27$ & $11.0(16.5)$ & $6.54-17.10$ & $1.0(1.4)$ & $0.08-3.83$ & $0.6(0.8)$ & $0.01-3.18$ \\
\hline \multicolumn{9}{|l|}{ Women $(n=114)$} \\
\hline Study sample ${ }^{a}$ & $14.0(16)$ & $8.24-21.79$ & $13.2(15)$ & $7.62-20.95$ & 0 & N/A & 0 & N/A \\
\hline Study population ${ }^{\mathrm{b}}$ & $14.2(14.7)$ & $8.30-22.21$ & $13.5(13.7)$ & $7.67-21.36$ & 0 & N/A & 0 & N/A \\
\hline \multicolumn{9}{|l|}{ Age $<65$ years $(n=174)$} \\
\hline Study sample ${ }^{a}$ & $13.2(23)$ & $8.57-19.17$ & $12.6(22)$ & $8.10-18.51$ & 0 & N/A & $0.6(1)$ & $0.01-3.16$ \\
\hline Study population ${ }^{\mathrm{b}}$ & $13.5(22.5)$ & $8.64-19.81$ & $13.0(21.6)$ & $8.21-19.25$ & 0 & $\mathrm{~N} / \mathrm{A}$ & $0.5(0.8)$ & $0.01-3.05$ \\
\hline \multicolumn{9}{|l|}{ Age $\geq 65$ years $(n=111)$} \\
\hline Study sample ${ }^{a}$ & $11.7(13)$ & $6.39-19.19$ & $9.0(10)$ & $5.10-17.19$ & $1.8(2)$ & $0.22-6.41$ & 0 & N/A \\
\hline Study population ${ }^{\mathrm{b}}$ & $11.9(10.3)$ & $6.52-19.43$ & $10.1(8.7)$ & $5.16-17.29$ & $1.7(1.4)$ & $0.18-6.19$ & 0 & N/A \\
\hline
\end{tabular}

To determine the prevalence of diabetic retinopathy in the population, data from the study sample were weighted for the age- and sex distributions of the local population. No cases of severe diabetic retinopathy or diabetic maculopathy were found

${ }^{a}$ Study sample: number of cases/observations in the study cohort

${ }^{\mathrm{b}}$ Study population: to determine the prevalence in the study population, data from the study sample were weighted for the age and sex distributions of the local population (region of Mainz/Mainz-Bingen, Germany)

representative of the background population [20]. In comparison, in a Danish population-based study, the prevalence of diabetic retinopathy in the population with type

Table 2 Effect of concomitant arterial hypertension, obesity and dyslipidaemia on the presence of diabetic retinopathy/ diabetic maculopathy in screening-detected type 2 diabetes

\begin{tabular}{llll}
\hline $\begin{array}{l}\text { Screening-detected type } 2 \\
\text { diabetes with the following } \\
\text { risk factors }\end{array}$ & $\begin{array}{l}\text { Diabetic retinopathy/ maculopathy } \\
\text { (all stages) }\end{array}$ & & \\
\cline { 2 - 4 } & $n(\%)$ & OR & $95 \% \mathrm{CI}^{\mathrm{a}}$ \\
\hline $\begin{array}{l}\text { Arterial hypertension } \\
\text { Yes }(n=202)\end{array}$ & $30(14.9)$ & 2.54 & $1.06-7.14$ \\
$\quad$ No $(n=83)$ & $6(7.2)$ & & \\
$\begin{array}{l}\text { Obesity } \\
\text { Yes }(n=146)\end{array}$ & $20(13.7)$ & 1.20 & $0.60-2.46$ \\
$\quad$ No $(n=139)$ & $16(11.5)$ & & \\
Dyslipidaemia & & & \\
$\quad$ Yes $(n=124)$ & $11(8.9)$ & 0.52 & $0.23-1.07$ \\
No $(n=159)$ & $25(15.7)$ & & \\
\hline
\end{tabular}

Age- and sex-adjusted ORs according to binary logistic regression analysis (including age, sex and one further variable, respectively)

${ }^{a}$ CIs based on the profile likelihood method
2 diabetes was $31.5 \%$ [21]. As in the GHS, no cases of severe non-proliferative or proliferative diabetic retinopathy were found. In the Diabetes Prevention Program (DPP) cohort, the prevalence of diabetic retinopathy in new-onset diabetes was $12.6 \%$ [22], whereas the median prevalence of diabetic retinopathy in newly diagnosed diabetes was $10.5 \%$ across 26 population-based studies (all from outside Europe) [23]. The lowest prevalence was found in African-American individuals $(1.5 \%)$ and the highest in a population from China (30.6\%). In the Hoorn study, the prevalence of diabetic retinopathy was higher in screening-detected type 2 diabetic individuals than in those newly diagnosed in general practice [19]. In the Beaver Dam Eye Study, the prevalence of sightthreatening diabetic retinopathy in screening-detected diabetes was only $2 \%$, and the authors therefore concluded that asymptomatic individuals discovered to have diabetes during epidemiological studies may not need immediate ophthalmoscopic examination at the time of their diagnosis because they have a relatively low risk of danger of visual loss at that time [24].

Association with $\mathrm{HbA}_{1 \mathrm{c}}$, hypertension and variables suggestive of the metabolic syndrome In the present study, 
the majority of participants with screening-detected type 2 diabetes and diabetic retinopathy had systemic hypertension. Approximately one half of the population with diabetic retinopathy were obese, and one third had dyslipidaemia. This observation is in line with earlier studies (that were not population-based) in which high levels of serum lipids and blood pressure were identified as risk factors for diabetic retinopathy [25]. In our study cohort of screening-detected type 2 diabetes, the presence of diabetic retinopathy was associated with neither the $\mathrm{HbA}_{1 \mathrm{c}}$ concentration nor the presence of arterial hypertension, obesity and dyslipidaemia. In the ADDITION study (screening in general practices), patients with diabetic retinopathy had significantly higher $\mathrm{HbA}_{1 \mathrm{c}}$ and systolic and diastolic blood pressures than those without retinopathy [20]. In a disease cohort of diabetic individuals in which systolic blood pressure and $\mathrm{Hb}_{\mathrm{A} 1 \mathrm{c}}$ concentrations were higher in those with than those without diabetic retinopathy, Spijkerman et al [26] did not find an association between $\mathrm{HbA}_{1 \mathrm{c}}$ levels and diabetic retinopathy in participants with screening-detected diabetes, but higher rates of arterial hypertension and dyslipidaemia were observed. In a study of the prevalence of and risk factors for diabetic retinopathy in people with newly diagnosed type 2 diabetes mellitus using Scottish national data, diabetic retinopathy was associated with male sex, systolic blood pressure, time to screening and obesity [27].

In terms of dyslipidaemia, intraretinal modified LDLs have been implicated in diabetic retinopathy, and it has been shown that extravascular modified LDLs may promote retinal pigment epithelium injury through oxidative stress, endoplasmic reticulum autophagy and apoptosis, whereas modified HDLcholesterol had protective effects [28, 29]. The same holds true for other novel risk markers such as serum apolipoprotein AI [30]. Unfortunately, these variables were not measured in the GHS.

There is controversy regarding the effect of screening on overall mortality. In a study by Jansson et al, no reduction in total mortality or cardiovascular disease outcomes was found in patients with type 2 diabetes who were detected by screening compared with those diagnosed clinically [31]. The Hoorn study, on the other hand, found that the mortality risk increased with increasing duration of diabetes [32]. In individuals whose diabetes is of short duration, the mortality risk could largely be attributed to other risk factors, whereas in those with a longer duration of diabetes, the elevated mortality risk was independent of cardiovascular risk factors.

Visual acuity in screening-detected diabetes In the present study, visual acuity was not impaired in the vast majority of participants, and no cases of severe non-proliferative diabetic retinopathy or maculopathy were observed. This finding was in line with other studies [23] and reflects the fact that type 2 diabetes may be asymptomatic even when end-organ damage is already detectable.

Limitations and perspectives One limitation of the present study is that non-mydriatic fundus photos were taken, and we were only able to take three fundus photos instead of the gold standard of seven. This limitation may have led to an underestimation of the prevalence of diabetic retinopathy because more peripheral lesions (e.g. microaneurysms) were not detected with this method. Nevertheless, it has recently been shown that $14 / 35$ studies on diabetic retinopathy have not applied pupil dilation [33]. Furthermore, it has been shown that even taking two $45^{\circ}$ photographic fields per eye is a promising alternative to the routine ophthalmologist's examination when screening for diabetic retinopathy. In a study by Stellingwerf et al, the sensitivity of two-field photography in identifying diabetic individuals with sightthreatening diabetic retinopathy was 95\% (specificity $99 \%$ ), and the sensitivity for detecting any diabetic retinopathy was $83 \%$ (specificity $88 \%$ ) [34].

Another limitation of this study has previously been published. For quality control purposes, we assessed the fundus images of 218 age- and sex-matched controls who did not have diabetes [17]. Approximately $10 \%$ of these control participants had retinal changes that would have been assessed as a mild stage of diabetic retinopathy. This can be attributed to the definition of diabetic retinopathy (i.e. that is already present when one microaneurysm is visible), to image quality and to other retinal microangiopathies.

A further limitation of the present study is that microalbuminuria was not assessed in the GHS. It would have been interesting to assess associations between microalbuminuria and diabetic retinopathy, especially because it has been shown that diabetic retinopathy is useful in diagnosing or screening for diabetic nephropathy in patients with diabetes and renal disease [35]. Furthermore, in a study on an elderly cohort of Icelanders ( $\geq 67$ years), over threequarters of cases of retinopathy occurred in individuals without diabetes, and a strong association was found between microalbuminuria and non-diabetic retinopathy [36]. Therefore, microalbuminuria should be tested in future population-based studies.

The results of this study have clearly highlighted the high number of individuals in the community who have undetected diabetes mellitus, and have indicated the need for screening for type 2 diabetes to detect early complications and prevent severe ophthalmic complications and visual impairment.

Acknowledgements We are indebted to all study participants and all members of the GHS who were involved in planning and conducting this study.

Funding The GHS is funded through the government of RhinelandPalatinate (Stiftung Rheinland-Pfalz für Innovation, contract AZ 
961-386261/733), the research programmes Wissen schafft Zukunft and Center for Translational Vascular Biology (CTVB) as well as the Center for Thrombosis and Hemostasis (CTH) of the Johannes GutenbergUniversity of Mainz, Germany, and its contract with Boehringer Ingelheim, PHILIPS Medical Systems and Novartis, including an unrestricted grant for the GHS. PSW and KAP are funded by the Federal Ministry of Education and Research (BMBF 01EO1003). PSW is principal investigator of the German Center for Cardiovascular Research (DZHK). TP is funded by the NIHR Biomedical Research Centre at Moorfields Eye Hospital NHS Foundation Trust and UCL Institute of Ophthalmology, UK. The funders had no role in the study design, data analysis, decision to publish or preparation of the manuscript.

Duality of interest The authors declare that there is no duality of interest associated with this manuscript.

Contribution statement Concept and design: KAP, PSW, NP, AM, $\mathrm{TP}$; data collection and analysis: PR, JL, TP; analysis and/or interpretation of data: KAP, JK, TP, KJL, NP; composition of the manuscript: KAP, AM; critical revision of the intellectual content: KAP, PR, JL, TP, JK, PSW, KJL, NP, AM; final approval of the version to be published: KAP, PR, JL, TP, JK, PSW, KJL, NP, AM. KAP and AM are the guarantors of this work.

\section{REFERENCES}

1. Sivaprasad S, Gupta B, Crosby-Nwaobi R, Evans J (2012) Prevalence of diabetic retinopathy in various ethnic groups: a worldwide perspective. Surv Ophthalmol 57:347-370

2. Olafsdottir E, Andersson DK, Dedorsson I, Svardsudd K, Jansson SP, Stefansson E (2016) Early detection of type 2 diabetes mellitus and screening for retinopathy are associated with reduced prevalence and severity of retinopathy. Acta Ophthalmol 94:232-239

3. Soto-Pedre E, Hernaez-Ortega MC, Pinies JA (2007) Duration of diabetes and screening coverage for retinopathy among patients with type 2 diabetes. Ophthalmic Epidemiol 14:76-79

4. (1991) Grading diabetic retinopathy from stereoscopic color fundus photographs-an extension of the modified Airlie House classification. ETDRS report number 10. Early Treatment Diabetic Retinopathy Study Research Group. Ophthalmology 98: 786-806

5. Porta M, Maurino M, Severini S et al (2013) Clinical characteristics influence screening intervals for diabetic retinopathy. Diabetologia $56: 2147-2152$

6. Goyder EC, Irwig LM (2000) Screening for Type 2 diabetes mellitus: a decision analytic approach. Diabet Med 17:469-477

7. Heintz E, Wirehn AB, Peebo BB, Rosenqvist U, Levin LA (2010) Prevalence and healthcare costs of diabetic retinopathy: a population-based register study in Sweden. Diabetologia 53: 2147-2154

8. Aspelund T, Thornorisdottir O, Olafsdottir E et al (2011) Individual risk assessment and information technology to optimise screening frequency for diabetic retinopathy. Diabetologia 54:2525-2532

9. van der Heijden AA, Walraven I, van't Riet E et al (2014) Validation of a model to estimate personalised screening frequency to monitor diabetic retinopathy. Diabetologia 57:1332-1338

10. Adriaanse MC, Snoek FJ, Dekker JM et al (2004) No substantial psychological impact of the diagnosis of Type 2 diabetes following targeted population screening: the Hoorn Screening Study. Diabet Med 21:992-998
11. Sawicki PT (2012) Screening for diabetes: hope and despair. Diabetologia 55:1568-1571

12. Forster AS, Forbes A, Dodhia H et al (2013) Non-attendance at diabetic eye screening and risk of sight-threatening diabetic retinopathy: a population-based cohort study. Diabetologia 56: 2187-2193

13. Kontopantelis E, Springate DA, Reeves D et al (2014) Glucose, blood pressure and cholesterol levels and their relationships to clinical outcomes in type 2 diabetes: a retrospective cohort study. Diabetologia

14. Kostev K, Rathmann W (2013) Diabetic retinopathy at diagnosis of type 2 diabetes in the UK: a database analysis. Diabetologia 56: $109-111$

15. Cignarelli M, De Cicco ML, Damato A et al (1992) High systolic blood pressure increases prevalence and severity of retinopathy in NIDDM patients. Diabetes Care 15:1002-1008

16. Lamparter J, Raum P, Pfeiffer N et al (2014) Prevalence and associations of diabetic retinopathy in a large cohort of prediabetic subjects: the Gutenberg Health Study. J Diabetes Complicat 28: 482-487

17. Raum P, Lamparter J, Ponto KA et al (2015) Prevalence and cardiovascular associations of diabetic retinopathy and maculopathy: results from the gutenberg health study. PLoS One 10:e0127188

18. Sabanayagam C, Liew G, Tai ES et al (2009) Relationship between glycated haemoglobin and microvascular complications: is there a natural cut-off point for the diagnosis of diabetes? Diabetologia 52: $1279-1289$

19. Spijkerman AM, Dekker JM, Nijpels G et al (2003) Microvascular complications at time of diagnosis of type 2 diabetes are similar among diabetic patients detected by targeted screening and patients newly diagnosed in general practice: the hoorn screening study. Diabetes Care 26:2604-2608

20. Bek T, Lund-Andersen H, Hansen AB, Johnsen KB, Sandbaek A, Lauritzen $T$ (2009) The prevalence of diabetic retinopathy in patients with screen-detected type 2 diabetes in Denmark: the ADDITION study. Acta Ophthalmol 87:270-274

21. Hove MN, Kristensen JK, Lauritzen T, Bek T (2004) The prevalence of retinopathy in an unselected population of type 2 diabetes patients from Arhus County, Denmark. Acta Ophthalmol Scand 82:443-448

22. (2007) The prevalence of retinopathy in impaired glucose tolerance and recent-onset diabetes in the Diabetes Prevention Program. Diabet Med 24:137-144

23. Ruta LM, Magliano DJ, Lemesurier R, Taylor HR, Zimmet PZ, Shaw JE (2013) Prevalence of diabetic retinopathy in Type 2 diabetes in developing and developed countries. Diabet Med 30: 387-398

24. Klein R, Klein BE, Moss SE, Linton KL (1992) The beaver dam eye study. Retinopathy in adults with newly discovered and previously diagnosed diabetes mellitus. Ophthalmology 99:58-62

25. Ding J, Wong TY (2012) Current epidemiology of diabetic retinopathy and diabetic macular edema. Curr Diab Rep 12: 346-354

26. Spijkerman AM, Adriaanse MC, Dekker JM et al (2002) Diabetic patients detected by population-based stepwise screening already have a diabetic cardiovascular risk profile. Diabetes Care 25: 1784-1789

27. Looker HC, Nyangoma SO, Cromie D et al (2012) Diabetic retinopathy at diagnosis of type 2 diabetes in Scotland. Diabetologia 55:2335-2342

28. Du M, Wu M, Fu D et al (2013) Effects of modified LDL and HDL on retinal pigment epithelial cells: a role in diabetic retinopathy? Diabetologia 56:2318-2328 
29. Fu D, Wu M, Zhang J et al (2012) Mechanisms of modified LDLinduced pericyte loss and retinal injury in diabetic retinopathy. Diabetologia 55:3128-3140

30. Sasongko MB, Wong TY, Nguyen TT, Shaw JE, Jenkins AJ, Wang JJ (2012) Novel versus traditional risk markers for diabetic retinopathy. Diabetologia 55:666-670

31. Jansson SP, Andersson DK, Svardsudd K (2016) Mortality and cardiovascular disease outcomes among 740 patients with new-onset Type 2 diabetes detected by screening or clinically diagnosed in general practice. Diabet Med 33:324-331

32. Spijkerman AM, Dekker JM, Nijpels G et al (2002) Impact of diabetes duration and cardiovascular risk factors on mortality in type 2 diabetes: the Hoorn Study. Eur J Clin Investig 32:924-930
33. Yau JW, Rogers SL, Kawasaki R et al (2012) Global prevalence and major risk factors of diabetic retinopathy. Diabetes Care 35:556-564

34. Stellingwerf C, Hardus PL, Hooymans JM (2001) Two-field photography can identify patients with vision-threatening diabetic retinopathy: a screening approach in the primary care setting. Diabetes Care 24:2086-2090

35. He F, Xia X, Wu XF, Yu XQ, Huang FX (2013) Diabetic retinopathy in predicting diabetic nephropathy in patients with type 2 diabetes and renal disease: a meta-analysis. Diabetologia 56: $457-466$

36. Gunnlaugsdottir E, Halldorsdottir S, Klein R et al (2012) Retinopathy in old persons with and without diabetes mellitus: the Age, Gene/Environment Susceptibility-Reykjavik Study (AGES-R). Diabetologia 55:671-680 